\title{
Association of Stillbirths with Maternal and Fetal Risk Factors in a Tertiary Care Hospital in South India
}

\author{
Rajshekher V. Mali (i), Anita Dalal $(\mathbb{D}$, Romana Khursheed $(\mathbb{D}$, and Aditya Gan \\ Department of Obstetrics and Gynecology, Jawaharlal Nehru Medical College, \\ KLE Academy of Higher Education and Research (Deemed to Be University), Belagavi-590010, Karnataka, India
}

Correspondence should be addressed to Romana Khursheed; romiarif@gmail.com

Received 28 April 2021; Revised 16 June 2021; Accepted 9 July 2021; Published 23 July 2021

Academic Editor: Yoshitsugu Chigusa

Copyright (c) 2021 Rajshekher V. Mali et al. This is an open access article distributed under the Creative Commons Attribution License, which permits unrestricted use, distribution, and reproduction in any medium, provided the original work is properly cited.

\begin{abstract}
Background. Birth of a fetus with no signs of life after a predefined age of viability is a nightmare for the obstetrician. Stillbirth is a sensitive indicator of maternal care during the antepartum and intrapartum period. Though there has been a renewed global focus on stillbirth as a public health concern, the decline in stillbirth rate (SBR) has not been satisfactory across the nations, with a large number of stillbirths occurring in the low- to middle-income countries (LMICs). Hence, the study was carried out to analyze maternal and fetal risk factors and their association with stillbirths in a tertiary care center in South India. Methods. This observational prospective study included pregnant women with stillbirth beyond 20 weeks of gestation or fetal weight more than 500 grams. Stillbirths were classified according to the simplified causes of death and associated conditions (CODAC) classification. Association between the risk factor and stillbirths was calculated with chi-square test and odds ratio with $95 \%$ confidence interval. Results. There were 171 stillbirths (2.97\%) among total 5755 births. The SBR was 29.71/1000 births. Risk factors such as preterm delivery (OR: 22.33, 95\% CI: 15.35-32.50), anemia (OR: 21.87, 95\% CI: 15.69-30.48), congenital malformation (OR: 11.24, 95\% CI: 6.99-18.06), abruption (OR: 10.14, 95\% CI: 6.43-15.97), oligohydramnios (OR: 4.88, 95\% CI: 3.23-7.39), and hypertensive disorder (OR: 3.01, 95\% CI: 2.03-4.46) were significantly associated with stillbirths. The proportion of intrapartum stillbirths was found to be $5(3 \%)$ among the study population. Conclusion. Highest prevalent risk factors associated with stillbirth are anemia and prematurity. Intrapartum stillbirths can be reduced significantly through evidence-based clinical interventions and practices in resource-poor settings. There is a need to provide and assure access to specialized quality antenatal care to pregnant women to control the risk factors associated with stillbirths.
\end{abstract}

\section{Background}

Stillbirth is defined generally as delivery of a fetus, following a predefined period of gestation and before complete expulsion from its mother, showing no signs of life and who cannot be resuscitated following birth [1]. In India, a fetus $\geq 20$ weeks of gestation with no signs of life is considered stillborn [2]. Stillbirth is the most prevalent adverse outcome of pregnancy. Death of a viable fetus is one of the most distressing events for the parents as well as for the obstetrician. Also, it is a sensitive marker of quality and equity of healthcare. The stillbirth rate (SBR) was $18.4 / 1000$ total births worldwide in 2015 [3]. The low- to middle-income countries (LMICs) contribute to $98 \%$ of stillbirths, and yearly, 592,100 stillbirths occur in India, with a rate of 22/ 1000 total births (World Health Organization (WHO)) $[4,5]$. The Every Newborn Action Plan (ENAP) in year 2014 has proposed a stillbirth rate target of 12 or fewer per 1000 births by year 2030 [6]. The 2019 ENAP progress report has depicted that $59 \%$ of the reporting countries will fail to achieve the stillbirth interim target of $14 / 1000$ or less by 2020 [6]. The annual reduction in stillbirths across the globe is $2 \%$, which is lesser than the decline in maternal mortality (3\%) and under-five child mortality (4.5\%) [7]. Though stillbirths comprise a large proportion of preventable deaths, it is challenging to determine their cause as the fetus is not directly observed when death occurs and the events prior to it. Furthermore, there is lag in knowledge in understanding 
the conditions and contexts before stillbirth occurs. The 2019 ENAP progress report recommends to intensify research into causes of stillbirth so that the results of these studies direct policy developments to reach the global target of stillbirth reduction.

Assigning a cause to the stillbirth and establishing its association with different maternal and fetal factors can help to prioritize interventions to improve birth outcomes in resource-poor areas. The present study attempted to identify the possible risk factors and their association with stillbirths in a tertiary care center in South India.

\section{Materials and Methods}

2.1. Study Setting. The present prospective, observational study was conducted from January 1, 2017, to December 31, 2017, at Karnataka Lingayat Education Academy of Higher Education and Research's Dr. Prabhakar Kore Charitable Hospital, attached to Jawaharlal Nehru Medical College, Belagavi, South India. It is a 1200-bed multispecialty hospital which is a referral center for the neighboring states. The labor ward is having total 64 beds and two operation theatres. Among these, 20 beds are obstetric intensive care beds. Annually, there are approximately 6000 deliveries. The labor ward is managed round the clock by a team of consultants, nurses, and postgraduates in obstetrics and gynecology (OBG), pediatrics, and anesthesiology departments. The institutional evidence-based protocols are used as guidelines for the management of high-risk cases.

\subsection{Study Participants}

2.2.1. Inclusion Criteria. All the women who were admitted to the labor ward and had stillbirth and consented for enrollment were included in the study. Written informed consent was obtained from all the study participants.

\subsubsection{Exclusion Criteria. The exclusion criterion was} gravidas having live births.

For the present work, ethical approval was obtained from the Institutional Ethics Committee of Jawaharlal Nehru Medical College of KLE Academy of Higher Education and Research, Belagavi, which certified that the study was performed in accordance with the ethical standards as laid down in the 1964 Declaration of Helsinki and its later amendments or comparable ethical standards.

2.3. Data Collection Procedure. The study proforma was discussed and verified by a group of obstetricians in a departmental review meeting. The pretesting of the study proforma was done before starting the data collection, and necessary changes were made and confirmed. The stillbirth data were recorded by a postgraduate medical student in the Obstetrics and Gynecology department, who was trained to get acquainted with the methodology of the research study. They were verified by an assigned professor in OBG.

The study participants were analyzed in terms of age, gravidity, and socioeconomic status (as per the 2016
Kuppuswamy scale for the urban population and the 2016 BG Prasad scale for the rural population) [8]. In the index pregnancy, details of antenatal checkups, medical illnesses, presence of obstetric complications, and other significant illnesses were evaluated. The gestational age was assigned as per the first trimester dating ultrasound or by Sonocare software if the dating scan was not available. Complete general physical examination/systemic examination and obstetric examination were conducted. Absence of fetal heart sound was confirmed by ultrasound examination. Mode of delivery and birth weights of fetuses and presence or absence of malformation were noted.

Every stillbirth was discussed in detail in perinatal death audit in the department of OBG of the institution, and cause of death was assigned based on history, clinical examination, and available investigations by a multidisciplinary team, consisting of a senior obstetrician and neonatologist. The cause of death was further classified as per the simplified CODAC, system of classification for stillbirths [9].

\subsection{Operational Definitions}

(i) Registered gravida: a woman who had 4 or more antenatal visits

(ii) Preterm stillbirth: stillbirth occurring before 37 completed weeks of gestation

(iii) Postterm stillbirth: stillbirth occurring after 42 weeks of gestation

(iv) Antepartum stillbirth: the intrauterine fetal demise occurred before the onset of labor

(v) Intrapartum stillbirth: the intrauterine fetal demise occurred during labor

(vi) Early stillbirth: stillbirths which occurred between 20 and 27 weeks and 6 days

(vii) Late stillbirth: stillbirths which occurred after 28 weeks

(viii) Stillbirth: it was defined as birth of a baby, with no signs of life occurring after 20 weeks of pregnancy or with $>500$ grams of fetal weight [9]

(ix) Extreme prematurity: birth before 28 weeks of gestation

\section{Results}

3.1. The Stillbirth Rate (SBR). A total of 171 stillbirths (2.97\%), with the stillbirth rate of 29.71/1000 births among 5755 total births, were observed.

3.2. The Sociodemographic and Clinical Presentation of the Participants. According to our results, 88 (51.46\%) and 83 (48.54\%) stillbirths were observed among the rural and urban population, respectively. The majority of stillbirths, 51 $(61.45 \%)$ and $36(40.91 \%)$ in the urban and rural population, respectively, belonged to class 3 (lower middle class in the Kuppuswamy scale and middle class in the modified BG Prasad scale). Among registered gravidas, 77 stillbirths 
(45\%) were noted, and 94 stillbirths (55\%) were noted in unregistered gravidas. Most of the stillbirths $(n=140)$ $(81.9 \%)$ were found in the women of 20-30 years age group. Stillbirths were more common in primigravida that is 78 stillbirths (45.61\%) followed by 43 stillbirths $(25.15 \%)$ in gravida-2, 32 stillbirths (18.71\%) in gravida-3, and 18 stillbirths $(10.53 \%)$ in gravida-4 and above. It was observed that 136 stillbirths $(79.53 \%)$ were delivered vaginally, and 35 stillbirths $(20.47 \%)$ were delivered through cesarean section (18 abruption, 6 eclampsia with end-organ damage, 5 previous LSCS, 3 fetal distress, 1 placenta previa, 1 rupture uterus, and 1 obstructed labor) (Table 1).

3.3. Stillbirths at Different Gestational Ages. According to Figure 1, a total of 45 stillbirths (26.32\%) occurred in women with the gestational age of 20-27 weeks and 6 days, 90 stillbirths (52.63\%) were noted at gestational age between 28 weeks and 36 weeks and 6 days period of gestation, 34 stillbirths (19.88\%) were seen at gestational age between 37 and 41 weeks and 6 days, and 2 stillbirths (1.17\%) occurred at gestational age more than 42 weeks. The total number of preterm (<37 weeks) stillbirths was 135 (78.94\%), followed by $34(19.88 \%)$ in term (37-41 weeks) and $2(1.17 \%)$ in postterm ( $\geq 42$ weeks) gestation (Table 1$)$.

3.4. Stillbirths in Antepartum and Intrapartum Period. The proportion of antepartum and intrapartum stillbirths is shown in Figure 2. There were 5 stillbirths (2.9\%) in intrapartum period and 166 stillbirths (97.07\%) in antepartum period.

3.5. The Distribution of Birth Weight among Stillbirths. It was observed to be as follows: the maximum number of stillbirths, that is, 66 (38.59\%), had birth weight between 500 and 999 grams, followed by 34 stillbirths (19.88\%) with birth weight between 1000 and 1499 grams.19 stillbirths (11.11\%) were observed in both 2000-2499 grams and 2500-2999 grams categories. Six stillbirths (3.51\%) were reported between 3000 and 3499 grams, and 3 stillbirths (1.75\%) were present between 3500 and 4000 grams birth weight (Figure 3).

3.6. Causes of Death and Associated Condition (CODAC). The common causes of death in the order of decreasing frequency were fetal causes $(50(29.24 \%))$, maternal conditions (42 (24.56\%)), placental causes (28 (16.37\%)), and congenital malformations (25 (14.62\%)), whereas unknown causes were 16 (9.36\%). Extreme prematurity, hypertensive disorders in pregnancy, abruption, and congenital malformations were present in 45 (26.32\%), 33 (19.3\%), 27 (15.79\%), and 25 (14.62\%), respectively (Table 2$)$.

3.7. Association of Stillbirths with Maternal and Fetal Risk Factors. Table 3 describes that preterm delivery, hypertensive disorders, anemia, abruption, congenital malformations, and oligohydramnios were significantly associated with stillbirths $(p<0.05)$. Through unadjusted odds ratio, it was noted that odds of stillbirths were 22.33 (95\% CI: 15.35-32.50) times higher for the stillbirths with preterm delivery than the stillbirths with full-term delivery. An odd of stillbirths for the subjects with congenital anomalies was 11.24 (95\% CI: 6.99-18.06) times higher than the subjects without congenital anomalies. Also, it was observed that the odds of stillbirths were 3.01 (95\% CI: 2.03-4.46) times higher for the subjects with hypertensive disorder than subjects without hypertensive disorder. The odds of stillbirth were 10.14 (95\% CI: 6.43-15.97), 4.88 (95\% CI: 3.23-7.39), and 21.87 (95\% CI: 15.69-30.48) times higher for the subjects with abruption, oligohydramnios, and anemia than the subjects without abruption, oligohydramnios, and anemia, respectively.

\section{Discussion}

Among 5755 total births, 171 stillbirths (2.97\%) were observed. The SBR was calculated to be $29.71 / 1000$ births. According to a report in year 2018, the national range of SBR in India is 22 to $66 / 1000$ births [10]. It has decreased from $31.3 / 1000$ births in year 2010 to $23.8 / 1000$ births in year 2016 [11]. Given the current rate of annual decline in Indian stillbirths of $4.5 \%$, a higher diminution of $5.8 \%$, in the current rates, is vital to achieve the ENAP goal [11].

The stillbirths were more in unregistered women as compared to registered women, that is, 94 (54.97\%) and 77 (45.03\%) stillbirths, respectively, in the study setup. This is in concurrence to a study conducted by Rajagopal et al. (unregistered stillbirths $54.4 \%$ vs. registered stillbirths $45.5 \%$ ) [12]. The diagnosis and surveillance of high-risk pregnancies by skilled health personnel and prompt effective management of complications in registered gravidas can explain the low stillbirths in the registered women.

A majority of the stillbirths, that is 88 (51.46\%), were found in women from the rural population. Dandona et al. also found higher stillbirths of $62.4 \%$ in the rural population [13]. In India, though there is implementation of financial incentive programs for pregnant women attending health services, the quality of care in peripheral health facilities is usually compromised, and such women are referred late to tertiary care centers. It is observed that $20-30 \%$ of stillbirths result from suboptimal obstetric care [14]. The higher prevalence of stillbirths in the rural population suggests the need for improved obstetric care as well as availability of emergency services in the rural settings. The study revealed that most of the pregnant women who had stillbirth were from lower middle and middle socioeconomic status, $51(61.45 \%)$ and $36(40.91 \%)$, respectively. The socioeconomic status influences the pregnancy outcome and determines health-seeking behavior of the women in accessing antenatal, intranatal, and emergency obstetric care. The comparable higher stillbirths in lower and middle socioeconomic groups were noted in a study by Asalkar et al. $(43,33)[15]$.

Though advanced maternal age is a known risk factor for both increased perinatal morbidity and mortality, the majority of the stillbirths $(140(82 \%))$ were seen in the women 
TABLE 1: Sociodemographic and clinical characteristics of gravidas and their stillborn in Dr. Prabhakar Kore Charitable Hospital (January 1, 2017-December 31, 2017).

\begin{tabular}{|c|c|c|c|}
\hline Variable & Subvariable & Number & Percentage \\
\hline \multirow{2}{*}{ Residence } & Urban & 83 & 48.54 \\
\hline & Rural & 88 & 51.46 \\
\hline \multirow{5}{*}{ Urban population (Kuppuswamy scale) $(n=83)^{*}$} & I & 1 & 1.2 \\
\hline & II & 14 & 16.87 \\
\hline & III & 51 & 61.45 \\
\hline & IV & 13 & 15.66 \\
\hline & $\mathrm{V}$ & 4 & 4.82 \\
\hline Socioeconomic status & $R 1$ & 0 & 0 \\
\hline \multirow{4}{*}{ Rural population (BG Prasad scale) $(n=88)^{\#}$} & $R 2$ & 22 & 25 \\
\hline & $R 3$ & 36 & 40.91 \\
\hline & $R 4$ & 26 & 29.55 \\
\hline & R5 & 4 & 4.55 \\
\hline \multirow{2}{*}{ Antenatal care } & Registered & 77 & 45.03 \\
\hline & Unregistered & 94 & 54.97 \\
\hline \multirow{3}{*}{ Age distribution of women with stillbirths } & $<20$ years & 17 & 9.97 \\
\hline & $>20-<30$ years & 140 & 81.9 \\
\hline & $>30$ years & 14 & 8.19 \\
\hline \multirow{4}{*}{ Stillbirths in different gravidity } & Gravida-1 & 78 & 45.61 \\
\hline & Gravida-2 & 43 & 25.15 \\
\hline & Gravida-3 & 32 & 18.71 \\
\hline & $\geq$ Gravida- 4 & 18 & 10.53 \\
\hline \multirow{3}{*}{ Period of gestation at the time of stillbirth } & Preterm $(<37$ wk $)$ & 135 & 78.94 \\
\hline & Term (37-41 wk) & 34 & 19.88 \\
\hline & Postterm (>42wk) & 2 & 1.16 \\
\hline \multirow{2}{*}{ Mode of delivery in stillbirths } & Vaginal delivery & 136 & 79.53 \\
\hline & Cesarean section & 35 & 20.47 \\
\hline
\end{tabular}

* and "indicate the percentage taken among the total number of urban and rural populations, respectively, Kuppusamy scale-I: upper class; II: upper middle class; III: lower middle class; IV: upper lower class; V: lower class; BG Prasad scale-R1: upper class; $R 2$ : upper middle class; $R 3$ : middle class; $R 4$ : lower middle class; $R 5$ : lower class.

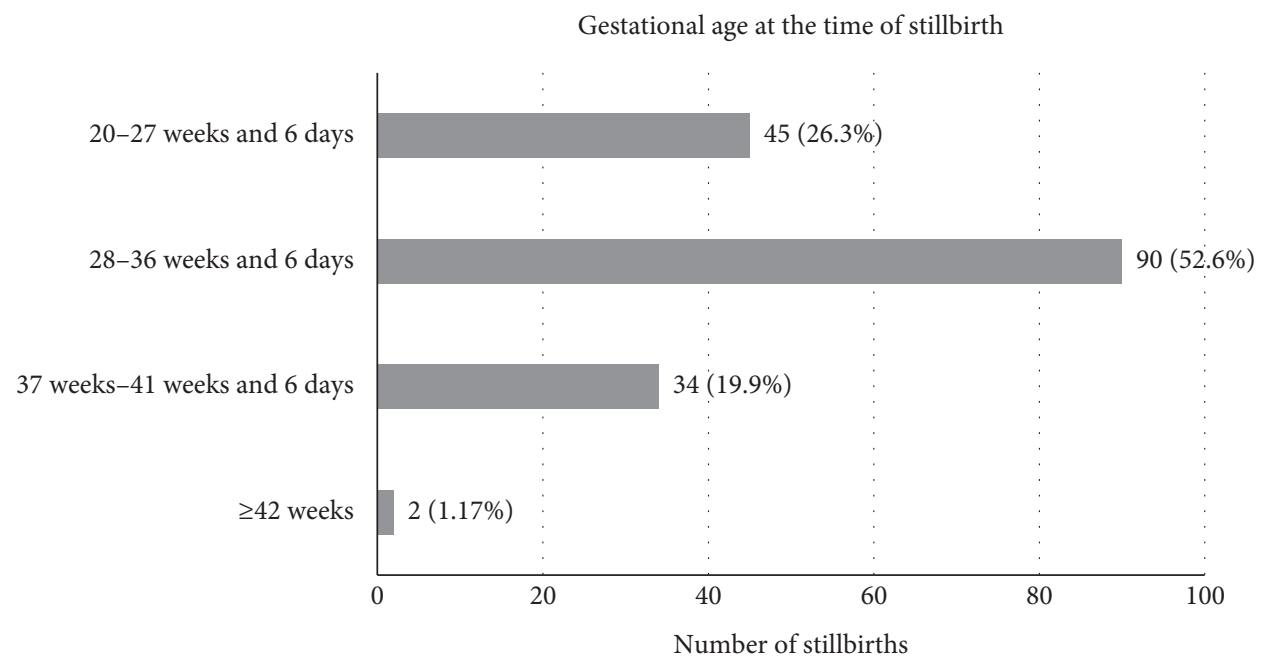

FiguRe 1: Number of stillbirths at different gestational ages.

between the age group of 20 and 30 years, similar higher rates were seen in a study conducted by Rajagopal et al. (71.4\%) [12]. In India, a higher prevalence of early marriage and completion of family before 35 years of life in women can explain the higher number of births and stillbirths in this age group. Lack of awareness about pregnancy-related complications and poor access to obstetric care facilities add fuel to this problem.

It was observed that stillbirths were more common in the primigravida (78 $(45.61 \%))$ among the study population. The 


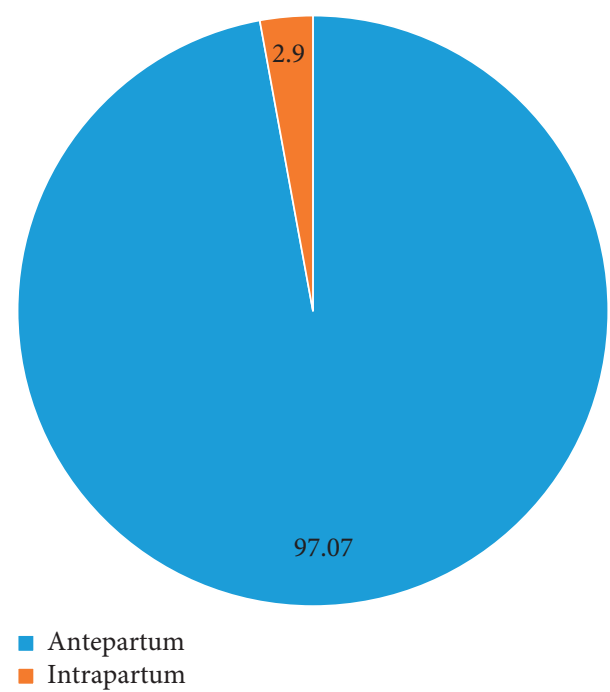

Figure 2: Proportion of stillbirths in the antepartum and intrapartum period.

relationship between gravidity and prevalence of the stillbirth is comparable to previous studies conducted by Asalkar et al. (primigravida 48.3\%) and Prasanna et al. (primigravida 49.8\%) [15, 16]. A study conducted by Saleem et al. also reported an increased risk of stillbirth in the first and after the fifth pregnancy [11]. In this study, almost half of stillbirths (90 (52.63\%)) were late stillbirths (28 weeks-36 weeks and 6 days), whereas one-fourth of stillbirths (45 (26.31\%)) were early stillbirths (20-27 weeks and 6 days).

The proportion of preterm stillbirths was 135 (78.94\%), and it was highest among stillbirths at different gestational ages in the study population. The higher number of late stillbirths is comparable to other studies such as Agbata et al. (81\%), Devi et al. (57\%), and Rajagopal et al. (75\%) $[10,12,17]$. The prevalence of low birth weight $(<2.5 \mathrm{~kg})$ including very low birth weight (1000-1499 grams) and extremely low birth weight (500-999 grams) was 143 $(83.62 \%)$ collectively in the study population. Similar results were seen by Sharma et al. (78.8\%) [9]. Fetal growth restriction and prematurity are important causes of low-birthweight stillbirths.

In this study, intrapartum deaths were 5 (3\%), and antepartum deaths were 166 (97\%). Lawn et al. estimated that intrapartum stillbirth rate was about $39 \%$ in middleincome countries [7]. Intrapartum stillbirths are quality indicators of a health institute. Low intrapartum stillbirths in this study reflected standard institutional protocol-based intrapartum care, close monitoring of the pregnant women during labor, availability of operation theatres and skilled personnel round the clock, and conduct of perinatal death audit for every stillbirth counted in the tertiary care center. Goldenberg et al. declared that, with every percentage rise in CS rate from 0 to $8 \%$, intrapartum stillbirths drop by $1.6 /$ 1000 births in LMIC [18].

It was found that cesarean sections were $35(20.47 \%)$ and vaginal deliveries were $136(79.53 \%)$ among stillbirths in this study. As the numbers of antepartum stillbirths were high in this study, such stillbirths are induced and delivered by the vaginal route unless there is a contraindication. For reducing antepartum stillbirths, there is a need to ensure access to quality care in terms of comprehensive interventions focusing on social, nutritional, and healthcare needs in developing nations as complications during the antepartum period are often associated with poor outcome of pregnancy. The causes of death were categorized as per the simplified CODAC system of classification among the study population. The common causes of death in the order of decreasing frequency were fetal causes (50 (29.24\%)), maternal conditions (42 $(24.56 \%))$, placental causes $(28(16.37 \%))$, and congenital malformations (25 (14.62\%)), whereas unknown causes were $16(9.36 \%)$. In a study conducted by Sharma et al., maternal conditions were $39.12 \%$ and unknown causes were $19.87 \%$ and ranked the highest in the causes of stillbirths in a tertiary care center in North India [9].

The prevalence of preterm delivery, hypertensive disorders of pregnancy, abruption, and congenital malformations with stillbirths was estimated to be 135 (78.94\%), 33 (19.29\%), 27 (15.79\%), and $25(14.62 \%)$ in the present study, and they were statistically significant $(p<0.05)$, with odds of stillbirth 22.33 (95\% CI: 15.35-32.50), 3.01 (95\% CI: 2.03-4.46), 10.14 (95\% CI: 6.43-15.97), and 11.24 (95\% CI: 6.99-18.06) times higher, respectively. Neogi et al. declared that the possibility of mothers with preterm delivery was 4.5 times higher of having stillbirth compared to full-term delivery [19]. The preterm stillbirths in this study include both spontaneous and induced preterm for different medical and obstetric complications. Significant association of the stillbirth with maternal hypertension was also noticed in many studies conducted in India and other countries [4, 20-22]. As abruption is commonly associated with hypertensive disorders of pregnancy, there is a need to follow stringent monitoring of women with preeclampsia, and timely intervention is crucial to reduce the burden of stillbirths associated with hypertensive disorders of pregnancy. Antepartum hemorrhage was reported as one of the top five causes of stillbirths in all income setting countries (low, middle, and high) [23]. Anemia and oligohydramnios were seen in 77 (45.03\%) and $30(17.54 \%)$ stillbirths in the study population with a statistically significant association $(p<0.0001)$ and higher odds of 21.87 and 4.88 compared to women without them. Altijani et al. also observed $35 \%$ higher odds of stillbirths with anemia compared with women who did not have anemia [4]. A higher odd for oligohydramnios was also seen in a study conducted by Zile et al. [24]. Congenital malformations were present in 25 stillbirths $(14.62 \%)(p=0.0005)$ and had 11.24 times higher odds of stillbirth in the present study. It was noted that most of the lethal anomalies were detected late in the pregnancy. Thus, timely anomaly scans at or before 20 weeks of gestation are highly recommended to detect lethal congenital anomalies early in pregnancy so that these are terminated within legal limits, and burden of stillbirths due to congenital anomalies is reduced.

Though fetal growth restriction, fetal distress, and diabetes mellitus in pregnancy are potential risk factors for stillbirths, they were not significantly associated with stillbirths in this study. Good intranatal care and institutional protocol-based management of high-risk pregnancy could 


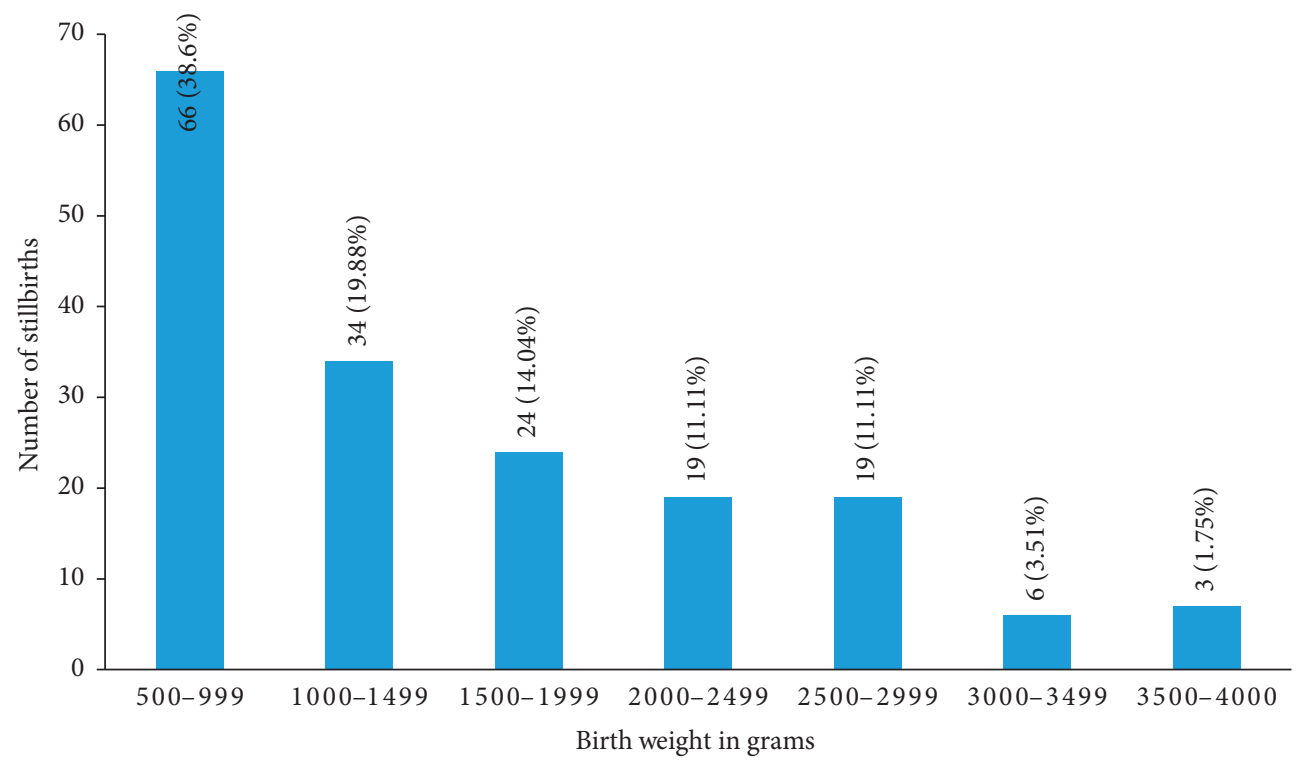

FIGURE 3: Birth weight (in grams) distribution in stillbirths.

TABle 2: Causes of stillbirths as per simplified CODAC in Dr. Prabhakar Kore Hospital (January 1, 2017-December 31, 2017).

\begin{tabular}{|c|c|c|c|}
\hline Cause of death & Subvariable & Number of stillbirths & Total stillbirths (\%) \\
\hline \multirow{2}{*}{ Infections $(n=2)(1.16 \%)$} & Chorioamnionitis & 1 & 0.58 \\
\hline & Toxoplasmosis & 1 & 0.58 \\
\hline \multirow{3}{*}{ Intrapartum $(n=5)(2.92 \%)$} & Fatal distress & 3 & 1.75 \\
\hline & Prolonged labor/obstructed labor & 1 & 0.58 \\
\hline & Rupture uterus & 1 & 0.58 \\
\hline \multicolumn{4}{|l|}{ Congenital anomaly $(n=25)(14.62 \%)$} \\
\hline \multirow{2}{*}{ Other fetal causes $(n=50)(29.24 \%)$} & Extreme prematurity & 45 & 26.32 \\
\hline & Hydrops of unknown origin & 5 & 2.92 \\
\hline \multirow{2}{*}{ Cord accidents $(n=3)(1.75 \%)$} & Loops & 2 & 1.17 \\
\hline & Cord prolapse & 1 & 0.58 \\
\hline \multirow{2}{*}{ Placental causes $(n=28)(16.37 \%)$} & Abruptio placenta & 27 & 15.79 \\
\hline & Placenta previa & 1 & 0.58 \\
\hline \multirow{5}{*}{ Maternal conditions $(n=42)(24.56 \%)$} & Hypertensive disorder & 33 & 19.3 \\
\hline & Preeclampsia & 26 & 15.2 \\
\hline & Eclampsia & 6 & 3.51 \\
\hline & Chronic hypertension & 1 & 0.58 \\
\hline & Diabetes & 7 & 4.09 \\
\hline \multicolumn{2}{|l|}{ Unknown } & 16 & 9.36 \\
\hline Total & 171 & 100 & \\
\hline \multirow{2}{*}{ Associated perinatal } & Small for gestational age & 30 & 17.54 \\
\hline & Multiple pregnancies & 2 & 1.17 \\
\hline \multirow{2}{*}{ Associated maternal } & Anemia & 77 & 45.03 \\
\hline & Oligohydramnios & 30 & 17.54 \\
\hline
\end{tabular}

be a reason for comparatively low stillbirths in these groups. Universal screening of pregnant women for diabetes and multidisciplinary management results in better compliance and reduced complications in diabetic pregnancy. We follow routine growth scan for all the antenatal females in the third trimester. This ensures early pick up of fetal growth restriction and low-birth-weight fetuses who further undergo rigorous follow-up with Doppler studies and need-based early termination.
4.1. Strengths and Limitations of the Study. Our study data are the first (to the best of our knowledge) to report the potential of reducing intrapartum stillbirths in a LMIC setup, with standard protocol-based institutional management of highrisk pregnancies. The real challenge in reducing overall stillbirths is to decrease the antepartum stillbirths. Since autopsies or other tests were not performed on stillborn babies, it is difficult to establish any causation in the unknown category. There are limitations in performing fetal autopsy in 
TABLe 3: Association of stillbirths with different maternal and fetal factors.

\begin{tabular}{|c|c|c|c|c|c|}
\hline $\begin{array}{l}\text { Maternal and fetal risk } \\
\text { factors }\end{array}$ & $\begin{array}{l}\text { Number of women with risk factors } \\
\text { among the total number of births } \\
\qquad(n=5755)\end{array}$ & $\begin{array}{l}\text { Number of stillbirths } \\
\text { with risk factors }\end{array}$ & Percentage & $\begin{array}{l}\text { Odds ratio } \\
(95 \% \mathrm{CI})\end{array}$ & $p$ value \\
\hline $\begin{array}{l}\text { Hypertensive disorders of } \\
\text { pregnancy }\end{array}$ & 454 & 33 & 19.29 & $3.01[2.03-4.46]$ & $<0.0001^{*}$ \\
\hline Abruption & 131 & 27 & 15.79 & $\begin{array}{c}10.14 \\
{[6.43-15.97]}\end{array}$ & $0.0005^{* \mathrm{MC}}$ \\
\hline Oligohydramnios & 269 & 30 & 17.54 & $4.88[3.23-7.39]$ & $<0.0001^{*}$ \\
\hline Anemia & 282 & 77 & 45.03 & $\begin{array}{c}21.87 \\
{[15.69-30.48]}\end{array}$ & $<0.0001^{*}$ \\
\hline Preterm delivery & 943 & 135 & 78.94 & $\begin{array}{c}22.33 \\
{[15.35-32.50]}\end{array}$ & $<0.0001^{*}$ \\
\hline $\begin{array}{l}\text { Premature rupture of } \\
\text { membranes }\end{array}$ & 682 & 15 & 8.77 & $0.73[0.43-1.25]$ & 0.2506 \\
\hline FGR & 915 & 30 & 17.54 & $1.16[0.78-1.74]$ & 0.458 \\
\hline Fetal distress & 70 & 03 & 1.75 & $1.52[0.47-4.87]$ & $0.7271^{\mathrm{MC}}$ \\
\hline $\mathrm{DM}$ & 372 & 7 & 4.09 & $0.63[0.29-1.35]$ & 0.2309 \\
\hline Congenital malformations & 111 & 25 & 14.62 & $\begin{array}{c}11.24 \\
{[6.99-18.06]}\end{array}$ & $0.0005^{* \mathrm{MC}}$ \\
\hline Maternal infection & 72 & 2 & 1.06 & $0.96[0.23-3.95]$ & $>0.99^{\mathrm{MC}}$ \\
\hline
\end{tabular}

MC: Monte Carlo's simulation used in chi-square test; ${ }^{*}$ indicates significance; FGR: fetal growth restriction; DM: diabetes mellitus.

stillbirths. Unwillingness by the parents due to financial and social concerns was identified as the most prevalent factor. Being a LMIC setup and due to financial constraints, certain advanced tests such as polymerase chain reaction (PCR) for infective pathology and cytogenetic analysis and MITS (minimally invasive tissue sampling) could not be performed. Future studies should focus on investigating their role in assessing the cause of death in stillbirths.

\section{Conclusion}

Preterm labor, anemia, congenital malformations, abruption, hypertensive disorder of pregnancy, and oligohydramnios were positively associated with stillbirths in this study. All the risk factors can be minimized by screening for early detection and prompt effective timely intervention in resource-poor settings. Perinatal death audit which includes thorough revision of stillbirths is a promising and practical quality improvement method that can be implemented in different settings and has potential to reduce the perinatal deaths. There is a need to regularize uniform protocols for antenatal and intranatal care in both urban and rural settings for a better neonatal outcome.

\section{Abbreviations}

CODAC: Cause of death and associated condition

ENAP: Every Newborn Action Plan

SBR: $\quad$ Stillbirth rate

WHO: World Health Organization

NICU: Neonatal intensive care unit

PCR: Polymerase chain reaction.

\section{Data Availability}

Preliminary data are available on request from the corresponding author. Data related to confidentiality of the subject are restricted.

\section{Conflicts of Interest}

The authors declare that they have no conflicts of interest.

\section{Authors' Contributions}

All authors contributed to the study conception and design. Material preparation, data collection, and analysis were performed by RM, AD, and AG. All the authors reviewed the manuscript and approved the final version of the manuscript.

\section{Acknowledgments}

The authors would like to acknowledge the study participants who gave consent for the participation.

\section{References}

[1] F. Tavares Da Silva, B. Gonik, M. McMillan et al., "Stillbirth: case definition and guidelines for data collection, analysis, and presentation of maternal immunization safety data," Vaccine, vol. 34, no. 49, pp. 6057-6068, 2016.

[2] Neonatal-Perinatal Database and Birth Defects Surveillance, Report of the Regional Review Meeting, New Delhi, India, 2014, https://apps.searo.who.int/PDS_DOCS/B5227.pdf.

[3] L. De Bernis, M. V. Kinney, W. Stones et al., "Stillbirths: ending preventable deaths by 2030," The Lancet, vol. 387, no. 10019, pp. 703-716, 2016.

[4] N. Altijani, C. Carson, S. S. Choudhury et al., "Stillbirth among women in nine states in India: rate and risk factors in study of 886,505 women from the annual health survey," $B M J$ Open, vol. 8, no. 11, Article ID e022583, 2018.

[5] H. Blencowe, S. Cousens, F. B. Jassir et al., "National, regional, and worldwide estimates of stillbirth rates in 2015, with trends from 2000: a systematic analysis," The Lancet Global Health, vol. 4, no. 2, pp. e98-e108, 2016.

[6] Ending Preventable Newborn Deaths and Stillbirths by 2030, http://www.unicef.org. 
[7] J. E. Lawn, H. Blencowe, P. Waiswa et al., "Stillbirths: rates, risk factors, and acceleration towards 2030," The Lancet, vol. 387, no. 10018, pp. 587-603, 2016.

[8] Z. Shaikh and R. Pathak, "Revised Kuppuswamy and B G Prasad socio-economic scales for 2016," International Journal Of Community Medicine And Public Health, vol. 4, no. 4, pp. 997-999, 2017.

[9] B. Sharma, G. Prasad, N. Aggarwal, S. Siwatch, V. Suri, and N. Kakkar, "Aetiology and trends of rates of stillbirth in a tertiary care hospital in the north of India over 10 years: a retrospective study," BJOG: An International Journal of $\mathrm{Ob}$ stetrics and Gynaecology, vol. 126, no. S4, pp. 14-20, 2019.

[10] K. S. Devi, N. Aziz, A. Gala, T. Surapaneni, N. H. Divya, and B. Hira, "Incidence of stillbirths and risk factors at a tertiary perinatal center in Southern India: retrospective observational study," International Journal of Gynecology and Reproductive Sciences, vol. 1, pp. 14-22, 2018.

[11] S. Saleem, S. S. Tikmani, E. M McClure et al., "Trends and determinants of stillbirth in developing countries: results from the Global Network's Population-Based Birth Registry," Reproductive Health, vol. 15, no. 1, pp. 100-130, 2018.

[12] V. M. Rajagopal, K. Betha, and G. S. Priya, "Classification of stillbirth by relative condition at death (re co De) at various trimesters of pregnancy: a rural tertiary teaching hospital based study," International Journal of Reproduction, Contraception, Obstetrics and Gynecology, vol. 6, no. 8, pp. 3550-3555, 2017.

[13] R. Dandona, G. A. Kumar, A. Kumar et al., "Identification of factors associated with stillbirth in the Indian state of Bihar using verbal autopsy: a population-based study," PLoS Medicine, vol. 14, no. 8, Article ID e1002363, 2017.

[14] V. Flenady, A. M. Wojcieszek, P. Middleton et al., "Stillbirths: recall to action in high-income countries," The Lancet, vol. 387, no. 10019, pp. 691-702, 2016.

[15] M. Asalkar, P. Gaikwad, and R. Pandey, "Perinatal morbidity and mortality due to preterm deliveries in a referral hospital, in rural India: a cross sectional study," Int J Reprod Contracept Obstetr Gynecol, vol. 2, 2013.

[16] N. Prasanna, K. Mahadevappa, R. Antaratani, and L. Lokare, "Cause of death and associated conditions of stillbirths," International Journal of Reproduction, Contraception, Obstetrics and Gynecology, vol. 4, no. 6, pp. 1970-1974, 2015.

[17] A. Agbata, J. Eze, C. Ukaegbe, and B. Odio, "A 4-year retrospective review of stillbirths at the federal teaching hospital, abakaliki, southeast Nigeria," African Journal of Medical and Health Sciences, vol. 16, no. 1, p. 19, 2017.

[18] R. L. Goldenberg, E. M. McClure, and C. M. Bann, "The relationship of intrapartum and antepartum stillbirth rates to measures of obstetric care in developed and developing countries," Acta obstetricia et gynecologica Scandinavica, vol. 86, no. 11, pp. 1303-1309, 2007.

[19] S. B. Neogi, J. Sharma, P. Negandhi, M. Chauhan, S. Reddy, and G. Sethy, "Risk factors for stillbirths: how much can a responsive health system prevent?" BMC Pregnancy and Childbirth, vol. 18, no. 1, pp. 1-0, 2018.

[20] M. Aminu, S. Bar-Zeev, S. White, M. Mathai, and N. van den Broek, "Understanding cause of stillbirth: a prospective observational multi-country study from subSaharan Africa," BMC Pregnancy and Childbirth, vol. 19, no. 1, p. 470, 2019.

[21] S. Sharma, H. Sidhu, and S. Kaur, "Analytical study of intrauterine fetal death cases and associated maternal conditions," International Journal of Applied and Basic Medical Research, vol. 6, no. 1, p. 11, 2016.
[22] T. Xiong, Y. Mu, J. Liang et al., "Hypertensive disorders in pregnancy and stillbirth rates: a facility-based study in China," Bulletin of the World Health Organization, vol. 96, no. 8, pp. 531-539, 2018.

[23] H. Reinebrant, S. Leisher, M. Coory et al., "Making stillbirths visible: a systematic review of globally reported causes of stillbirth," BJOG: An International Journal of Obstetrics \& Gynaecology, vol. 125, no. 2, pp. 212-224, 2018.

[24] I. Zile, I. Ebela, and I. Rumba-Rozenfelde, "Maternal risk factors for stillbirth: a registry-based study," Medicina, vol. 55, no. 7 , p. $326,2019$. 